\title{
Epicardial Fat Tissue Thickness and Omentin in Patients with Atrial Fibrillation
}

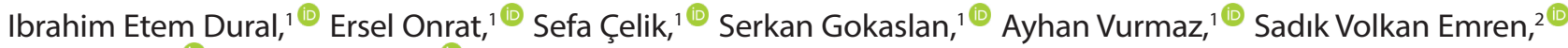 \\ Zafer Yalım, ${ }^{10}$ Alaeddin Avşar ${ }^{10}$ \\ Afyonkarahisar Health Sciences University, ${ }^{1}$ Afyonkarahisar - Turkey \\ Izmir Katip Celebi University, ${ }^{2}$ Izmir - Turkey
}

\section{Abstract}

Background: Although electrical and structural remodeling has been recognized to be important in the pathophysiology of atrial fibrillation, the mechanisms underlying remodeling process are unknown. There has been increasing interest in the involvement of inflammatory molecules and adipokines released from the epicardial fat tissue in the pathophysiology of atrial fibrillation.

Objectives: In our study, we aimed to investigate the relationship of atrial fibrillation with increased epicardial adipose tissue, inflammatory molecules released from this tissue and omentin.

Methods: Thirty-six patients who were followed up with a diagnosis of permanent AF at the cardiology outpatient clinic 33 individuals without atrial fibrillation (controls) were included in the study. Epicardial adipose tissue thickness of patients was measured by echocardiography. Serum omentin, IL 6, IL 1 beta, TNF alpha and CRP levels were measured. Man-Whitney $U$ test was performed for comparisons and significance was established at $5 \%$ $(\mathrm{p}<0.05)$.

Results: Epicardial adipose tissue thickness was significantly greater in the patient group (6mm [4-5.5]) than controls (4mm [3-5.5]) ( $\mathrm{p}<0.001)$. No significant difference was found in the concentrations of omentin or inflammatory molecules between the groups.

Conclusion: No relationship was found between atrial fibrillation and serum levels or omentin or inflammatory markers. A relationship between epicardial adipose tissue thickness measured by echocardiography and atrial fibrillation was determined.

Keywords: Atrial Fibrillation; Omentin, Inflammation; Intelectin 1; Pericardium; Adipose Tissue; Molecules.

\section{Introdução}

Atrial fibrillation (AF) is a severe arrhythmic condition with high mortality and morbidity. AF may have serious complications, and its incidence is increasing day by day. According to current guidelines, there are five types of AF - first diagnosed AF, paroxysmal AF, persistent $\mathrm{AF}$, long-standing persistent $\mathrm{AF}$, and permanent AF. ${ }^{1}$ Permanent AF is used when a joint decision by the patient and clinician has been made to no longer pursue a rhythm control strategy. ${ }^{1}$ Because of its long duration, permanent AF is more suitable for pathophysiological studies than other types of the disease. ${ }^{2}$
Due to the increasing incidence of AF, new treatment modalities have been developed and implemented. However, the pathophysiology of the disease has not been fully elucidated. ${ }^{3}$ In recent years, it has been suggested that that epicardial fat tissue, adipokines and inflammatory molecules released from this tissue may play a role in the pathophysiology of $\mathrm{AF}^{4-6}$

Epicardial adipose tissue is a visceral adipose tissue where molecules associated with inflammation and atherosclerosis are released. ${ }^{7}$ As the epicardial adipose tissue thickness increases, the levels of proinflammatory molecules such as interleukin -6 (IL-6) and tumor necrosis 
factor alfa (TNF alpha ) also increase in the tissue. ${ }^{8}$ We also know that adipokines such as adiponectin, leptin, and omentin are also released from epicardial adipose tissue like other visceral adipose tissues.,10 These proinflammatory molecules and adipokines are thought to be related to $\mathrm{AF}$.

There are many studies on the relationship of inflammation and inflammatory molecules with AF and the contribution of the inflammatory process to the formation of AF. ${ }^{11}$ Atrial fibrosis, which is an important factor in the formation of AF, is thought to develop as a result of inflammation in the atrial myocardium. Especially in patients with persistent and permanent $\mathrm{AF}$, higher $\mathrm{C}$-reactive protein (CRP) levels indicate this inflammatory process as compared with patients with paroxysmal AF and individuals with sinus rhythm. ${ }^{12}$ High detection of IL-1, IL-6, and TNF alpha in patients with AF supports this view. In addition, the higher levels of TNF alpha in patients with chronic AF and valvular AF compared to patients with paroxysmal AF indicates its relationship with chronic inflammation and atrial fibrosis. ${ }^{13}$

Omentin is an adipokine mostly released from visceral adipose tissue. ${ }^{14}$ Its anti-inflammatory effects have been shown in experimental studies, and the adipokine has also been detected in epicardial adipose tissue. ${ }^{10-13}$ The relationship of omentin released from epicardial adipose tissue with cardiovascular diseases has been shown in various studies. ${ }^{15}$ Serum levels of omentin, which suppresses the inflammatory process by multiple pathways, are low in some cardiovascular diseases. ${ }^{16}$ Omentin is thought to play a role in the pathophysiology of AF, although there are few studies on this topic. ${ }^{17}$

Thus, in the presen study, we aimed to show the relationship of AF with inflammatory molecules and omentin.

\section{Methods}

\section{Study population}

Thirty-six patients who were followed up with a diagnosis of permanent AF at the cardiology outpatient clinic were included in the study. In addition, diagnosis of permanent AF was applied to those cases when both physician and patient decided to accept the presence of AF at least one year before. Thirty-three individuals attending the cardiology outpatient clinic, who had sinüs rhythm (electrocardiography, ECG) were included in the control group. They didn't have atrial fibrillation in the ECG Holter monitoring.

Patients with mitral stenosis, moderate or severe mitral insufficiency, mechanical mitral valve, hyperthyroidism, and those who underwent AF ablation were excluded. Patients with cancer, autoimmune disease, connective tissue, and inflammatory bowel disease were also excluded because of the association of these conditions with elevated inflammatory biomarkers.

All subjects underwent conventional echocardiography. Left ventricular end-diastolic and end-systolic diameters, posterior wall and septal thickness, aortic root, left atrial dimension, ejection fraction, aortic and mitral valve maximum and mean gradients, systolic pulmonary artery pressure, and epicardial fat tissue thickness were measured. Ejection fraction was calculated with the Teichholz M-mode formula: (EDV-ESV)/EDVx100. EDV:[7/(2.4+End diastolic diameter(EDd)) $] \times E^{2}{ }^{3}$; ESV:[7/(2.4+ End systolic diameter (ESd))]xESd ${ }^{3}$

$\mathrm{EDV}=$ end-diastolic volume; $\mathrm{ESV}=$ end-systolic volume; $\mathrm{EDd}=$ end-diastolic diameter; $\mathrm{Esd}=$ end-systolic diameter

The local ethics committee approved the study protocol (approval date and number 01/29/2015 and 2015/02-30). The study complies with the Declaration of Helsinki.

\section{Measurement of epicardial fat thickness}

Echocardiographic examinations of all patients and the control group were performed with the help of the GE M5Sc-D probe of the General Electric Medical Systems Vivid E9 Xdclear device.

Transthoracic echocardiographic images of the patients were obtained with a 2.5-3.5 $\mathrm{MHz}$ transducer from parasternal and apical windows. Echocardiographically, epicardial fat thickness was mesaured on the parasternal long-axis and parasternal short-axis images while the patient was lying in the left lateral decubitus position. The area and thickness of epicardial fat between the outer layer of the free wall of the right ventricle and the visceral layer of the pericardium was measured at the end of the systole. Echocardiographic fat tissue thickness measurements were taken in three separate cycles to obtain more accurate values. All echocardiographic and Doppler studies were carried out by the same observer. 


\section{Statistical analysis}

Statistical analysis was conducted using the SPSS software version 18.0 (SPSS, Inc., Chicago, Illinois). Continuous variables with normal distribution were described as mean and standard deviation, and median and interquartile ranges were used for continuous variables not normally distributed. Categorical variables were presented as percentage.

The Kolmogorov-Smirnov test was used to test the normality of these data distribution. The chi-square test was used to compare nominal and categorical variables. Parametric data were compared using the Student's t-test and non-parametric data were compared using the Mann-Whitney U test. A two-sided p-value $<0.05$ was considered statistically significant. We calculated the sample size based on a statistical power of 0.95 , twosided alpha error of 0.05 , with an odds ratio (OR) of $1,{ }^{17}$ using the Gpower software v3.1.9.4 (Erdfelder, Faul, \& Buchner, 1996).

\section{Results}

Mean age was $68.5 \pm 15$ years in the patient group and $62.8 \pm 10.1$ years in the control group. In addition, $58 \%(21)$ of the patient group and $51 \%$ (17) of the control group were women. The use of antiplatelet agents was different in the patient and control groups. Demographic characteristics of individuals in the study are summarized in Table 1.

Epicardial adipose thickness and left atrial size were significantly greater in patients with $\mathrm{AF}$ than in the control group. Figure 1 shows the graph comparing epicardial adipose thickness.

Serum TNF-alpha, high-sensitivity CRP, IL-1 beta, IL-6 and omentin levels were not statistically different between the two groups (Figure 2 and Table 2).

Table 1 - Baseline clinical characteristics of the study participants

\begin{tabular}{|c|c|c|c|}
\hline Characteristics & $\begin{array}{l}\text { AF group } \\
(n=36)\end{array}$ & $\begin{array}{c}\text { Control group } \\
\qquad(n=33)\end{array}$ & $\mathbf{p}$ \\
\hline Age, years & $68.5 \pm 15$ & $62.8 \pm 10,1$ & 0.068 \\
\hline Male, n (\%) & $21(\% 58)$ & $17(\% 51)$ & 0.666 \\
\hline Body mass index (kg/m2) & $28.6 \pm 6.4$ & $27.8 \pm 4,1$ & 0.535 \\
\hline Coronary artery disease $\mathrm{n}(\%)$ & $10(\% 28)$ & $12(\% 36)$ & 0.445 \\
\hline Heart Failure n (\%) & $4(\% 11)$ & $5(\% 15)$ & 0.728 \\
\hline Hypertension n (\%) & $19(\% 53)$ & $18(\% 54)$ & 0.883 \\
\hline Diabetes mellitus n (\%) & $9(\% 25)$ & $7(\% 21)$ & 0.710 \\
\hline Dyslipidemia n (\%) & $9(\% 25)$ & $15(\% 45)$ & 0.075 \\
\hline Stroke n $(\%)$ & $1(\% 3$ & 0 & \\
\hline \multicolumn{4}{|l|}{ Drugs } \\
\hline Beta blocker n (\%) & $18(\% 50)$ & $12(\% 36)$ & 0.254 \\
\hline ACEi/ARB n (\%) & $21(\% 64)$ & $20(\% 56)$ & 0.495 \\
\hline Statin n $(\%)$ & $10(\% 28)$ & $5(\% 45)$ & 0.127 \\
\hline ASA n (\%) & 0 & $13(\% 39)$ & \\
\hline Oral anticoagulant $\mathrm{n}(\%)$ & $35(\% 97)$ & 0 & \\
\hline Oral antidiabetic n (\%) & $8(\% 22)$ & $8(\% 24)$ & 0.843 \\
\hline MRA n (\%) & $6(\% 17)$ & $4(\% 12)$ & 0.592 \\
\hline Diuretic n (\%) & $10(\% 28)$ & $15(\% 45)$ & 0.127 \\
\hline
\end{tabular}




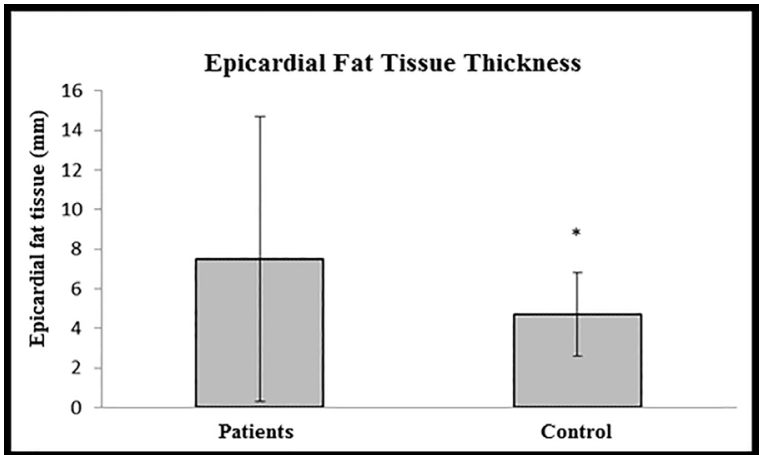

Figure 1 - Measurement of epicardial fat tissue thicknesses in patients $(n=36)$ and controls $(n=33)$.

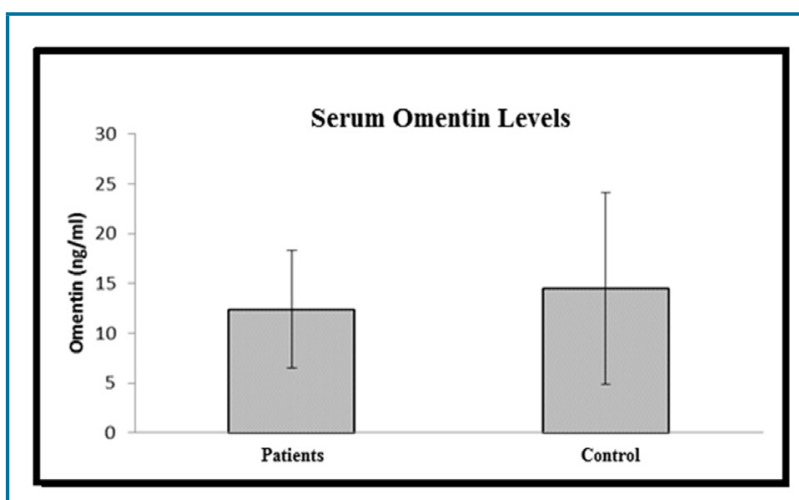

Figure 2 - Serum omentin levels in patients $(\mathrm{n}=36)$ and controls $(n=33)$.

Table 2 - Serum inflammatory molecules and serum omentin levels in the study groups

\begin{tabular}{lccc}
\hline Characteristics & $\begin{array}{c}\text { AF group } \\
(\mathbf{n}=36)\end{array}$ & $\begin{array}{c}\text { Control group } \\
(\mathbf{n}=33)\end{array}$ & $\mathbf{p}$ \\
\hline Inflammatory molecules & $27.635(20.658-30.813)$ & $27.935(21.723-27.935)$ & 0.959 \\
\hline TNF-alfa $(\mathrm{pg} / \mathrm{mL})$ & $6.69(2.34-15.21)$ & $6.53(3.2025-11.52)$ & 0.498 \\
\hline Hs-CRP $(\mathrm{mg} / \mathrm{mL})$ & $8.61(6.26-12.22)$ & $9.6(7.145-11.735)$ & 0.556 \\
\hline IL-1 beta $(\mathrm{pg} / \mathrm{mL})$ & $3.64(2-8.955)$ & $2.37(1.4825-4.2575)$ & 0.343 \\
\hline IL-6 $(\mathrm{pg} / \mathrm{mg})$ & $13.51(6.785-20.413)$ & $11.8(7.425-18.145)$ & 0.555 \\
\hline Omentin $(\mathrm{ng} / \mathrm{mL})$ & & & \\
\hline $\begin{array}{l}P \text { value }<0.05 \\
\text { TNF-alfa: } \text { tumor necrosis factor alpha, Hs-CRP: } \text { high-sensitive c-reactive protein, IL-1 beta: } \text { interleukin 1 beta, IL 6: interleukin } 6\end{array}$ \\
\hline
\end{tabular}

\section{Discussion}

In recent years, new risk factors for $\mathrm{AF}$ have been identified. Some of these new risk factors are obesity, and increased epicardial fat tissue, cytokines and inflammatory factors. Epicardial fat tissue is thought to be more specific than obesity in determining AF risk. Therefore, the relation of inflammatory molecules released from epicardial adipose tissue and adipokines with AF will provide a better understanding of the pathophysiology of the disease. ${ }^{18,19}$ In our study, we investigated serum levels of inflammatory molecules thought to be released from epicardial adipose tissue and of omentin, which has been poorly addressed.

The thickness of the epicardial fat tissue is related to the biological activity of the tissue, with greater release of cytokines from thick tissues..$^{20}$ There is strong evidence that cytokines released from epicardial fat tissue are effective in the pathogenesis of heart disease. Tissue studies have shown that epicardial fat tissue of patients with ischemic heart disease secretes high levels of interleukin 6, tumor necrosis factor-alpha and monocyte chemotactic factor 1 , and local concentrations of these cytokine are significantly higher than in systemic circulation. These results suggest that cytokines released from epicardial fat tissue act in the cardiac region. ${ }^{21-25}$

Our finding of increased epicardial adipose thickness in patients with AF was consistent with the literature, and supports the relationship between epicardial adipose tissue and AF.

- Inflammation is one of the new risk factors for AF, and the source of inflammatory cytokines secreted in cardiac diseases seems to be the epicardial fat tissue. ${ }^{19}$ This is supported by the increased frequency 
of AF in conditions of intense myocardial inflammation, such as pericarditis, myocarditis and cardiac surgery. It is known that atrial fibrosis, which occurs at the end of inflammation, plays an important role in the development of AF. Increased B-type natriuretic peptide (BNP) due to myocyte stretch and increasing high-sensitive troponin levels due to myocyte damage caused by inflammation and fibrosis supports this pathophysiological pathway. High BNP levels have been associated with ischemic complications, and high troponin levels have been associated with cardiovascular mortality in patients with $\mathrm{AF}^{22,23}$

- Inflammatory biomarkers detected in the atrial tissue reinforce the idea of the relationship between inflammation and AF. ${ }^{19}$ In atrial biopsies taken during cardiac surgery, high-sensitive CRP, IL-1, IL-6 and TNF alpha levels indicated an inflammatory process. High serum levels of these molecules, especially in patients with permanent $\mathrm{AF}$, have been reported in previous studies. ${ }^{11,18,19}$ In addition, these biomarkers have been investigated in disease prediction and evaluation of prognosis, ${ }^{11,20}$ and in treatment response in patients receiving beta-blocker therapy. ${ }^{24}$

- We could not find a significant difference in the levels of inflammatory markers between patients' and control groups in our study. The most important reason for this may be the inclusion of patients with inflammationrelated diseases such as coronary artery disease, diabetes and hypertension. Thus, the inclusion of patients from the general population rather than selected patients may have affected the outcomes in this direction. This is supported by the fact that inflammatory molecule levels were not normally distributed.

- Omentin is an anti-inflammatory adipokine released substantially from the epicardial and omentum fat tissues. ${ }^{14}$ Although omentin is secreted from the omentum, blood levels of this cytokine fall in obesity. In contrast, the relationship between epicardial fat tissue and blood omentin level are not fully clarified. Omentin has anti-inflammatory properties and suppresses inflammation through several biochemical pathways in experimental studies. ${ }^{25}$

- We thought that the decisive effect of inflammation and increased epicardial fat tissue in the development of AF was by means of omentin release. Epicardial adipose tissue, which is increased in patients with $\mathrm{AF}$, gradually progresses to adipose tissue dysfunction called adiposopathy. This, in turn, reduces the release of the anti-inflammatory cytokine omentin, ${ }^{26}$ resulting in inflammation. ${ }^{27}$ This inflammation is thought to be one of the causes of AF. ${ }^{18}$

- In our study, there was no significant difference between the two groups for omentin. We confirmed our hypothesis that increased epicardial fat tissue is the basis of AF, with involvement of inflammation and adipokines; however, we could not demonstrate the implication of omentin or biomarkers in it. We have shown that high body mass index is associated with increased epicardial fat tissue thickness, which may explain the association between obesity and AF.

- Our study is the second in the literature to examine the association of serum omentin levels with AF. Tao et al., ${ }^{17}$ showed a negative correlation between serum omentin levels and AF; the adipokine levels were significantly lower in patients with AF. However, in our study, this significant difference was not observed. One of the reasons for this can be that the larger sample size in their study. Also, Tao et al., ${ }^{17}$ did not include patients with chronic diseases. In our study, patients with coronary artery disease, diabetes mellitus, hypertension, and heart failure were included, with no statistical difference in the two groups.

- We also believed that adiposopathy, related to increased epicardial fat tissue, may cause AF through inflammation and omentin. However, in our study, adiposopathy was not detected in patients with increased epicardial fat tissue. We suggest that further studies be done to elucidate the pathophysiology of adiposopathy by different imaging and laboratory techniques.

\section{Study limitations}

The small numbers of patients and control subjects is the most important limitation of our study. Another limitation is the presence of diseases that could affect omentin and inflammatory biomarker levels.

\section{Conclusion}

Our study showed that increased epicardial fat tissue played an important role in $\mathrm{AF}$, and that it had a positive relationship with body mass index. We could not establish the relationship of omentin and inflammation with AF.

\section{Potential Conflict of Interest}

No potential conflict of interest relevant to this article was reported. 


\section{Sources of Funding}

This study was funded by Turkish Society of Cardiology.

\section{Study Association}

This study is not associated with any thesis or dissertation work.

\section{Ethics approval and consent to participate}

This study was approved by the Ethics Committee of the Afyon Health Science University under the protocol number 2015/02-30. All the procedures in this study were in accordance with the 1975 Helsinki Declaration, updated in 2013. Informed consent was obtained from all participants included in the study.

\section{Author contributions}

Conception and design of the research: Onrat E. Acquisition of data: Dural İE. Analysis and interpretation of the data: Emren SV. Statistical analysis: Vurmaz A. Writing of the manuscript: Dural İE. Critical revision of the manuscript for intellectual content: Avşar A.

\section{References}

1. January CT, Wann LS, Alpert JS, Calkins H, Cleveland JC Jr, Cigarroa JE. et al. AHA/ACC/HRS Guideline for the Management of Patients With Atrial Fibrillation: Executive Summary: A Report of the American College of Cardiology/American Heart Association Task Force on Practice Guidelines and the Heart Rhythm Society. J Am Coll Cardiol. 2014;64(21):e1-76. doi: 10.1016/j.jacc.2014.03.022.

2. Heijman J, Voigt N, Nattel S, Dobrev D. Cellular and molecular electrophysiology of atrial fibrillation initiation, maintenance, and progression. Circ Res. 2014;114(9):1483-9. doi:10.1161/ CIRCRESAHA. 114.302226

3. Alpert, J.S. Petersen, P. Godtfredsen, J. Atrial fibrillation: natural history, complications, and management. Ann Rev Med. 1988;39:4152. DOI: $10.1146 /$ annurev.me.39.020188.000353

4. Venteclef N, Guglielmi V, Balse E, Gaborit B , Cotillard A, Atassi F, et al. Human epicardial adipose tissue induces fibrosis of the atrial myocardium through the secretion of adipo-fibrokines. Eur Heart J. 2015;36(13):795-805a. DOI: 10.1093/eurheartj/eht099

5. Leggio M, Severi P, D'emidio S, Mazza A. Epicardial adipose tissue and atrial fibrillation: The other side of the coin. Anatol J Cardiol. 2017; 17(5):415-6. DOI: 10.14744/AnatolJCardiol.2017.7752

6. Hatem SN. Is epicardial adipose tissue an epiphenomenon or a new player in the pathophysiology of atrial fibrillation?. Arch Cardiovasc Dis. 2014;107(6-7):349-52. DOI: 10.1016/j.acvd.2014.06.002

7. Al Chekakie MO, Welles CC, Metoyer R, Ibrahim A, Shapira AR, Cytron J, et al. Pericardial fat is independently associated with human atrial fibrillation. J Am Coll Cardiol. 2010 Aug 31;56(10):784-8. DOI: 10.1016/j.jacc.2010.03.071

8. Mazurek T, Kiliszek M, Kobylecka M, Skubisz-Głuchowska J, Kochman J, Filipiak K, et al. Relation of proinflammatory activity of epicardial adipose tissue to the occurrence of atrial fibrillation. Am J Cardiol. 2014;113(9):1505-8. doi: 10.1016/j.amjcard.2014.02.005.

9. Ermakov S, Azarbal F, Stefanick ML, LaMonte MJ, Li W, Tharp KM,et al. The associations of leptin, adiponectin and resistin with incident atrial fibrillation in women. Heart. 2016 Sep 1;102(17):1354-62. doi: 10.1136/heartjnl-2015-308927.

10. Fain JN, Sacks HS, Buehrer B, Bahouth SW, Garrett E, Wolf RY, e al. Identification of omentin mRNA in human epicardial adipose tissue: comparison to omentin in subcutaneous, internal mammary artery periadventitial and visceral abdominal depots. Int J Obes (Lond) 2008;32(5):810-5

11. Scott L Jr, Li N, Dobrev D. Role of inflammatory signaling in atrial fibrillation. Int J Cardiol. 2019 Jul 15;287:195-200. doi: 10.1016/j. ijcard.2018.10.020.

12. Chung MK, Martin DO, Sprecher D, Wazni O, Kanderian A, Carnes CA, et al. C-reactive protein elevation in patients with

atrial arrhythmias: inflammatory mechanisms and persistence of atrial fibrillation. Circulation. 2001;104(24):2886-91. doi: 10.1161/ hc4901.101760.

13. Wang CH, Hu DY, Tang CZ, Wu MY, Mei YQ, Zhao JG, et al. Changes of interleukin-1beta and tumor necrosis factor-alpha of right atrial appendages in patients with rheumatic valvular disease complicated with chronic atrial fibrillation. Zhonghua Xin Xue Guan Bing Za Zhi. 2005 Jun;33(6):522-5. Chinese. PMID: 16053785.

14. Yang R, Xu A, Pray J, Hu H, Jadhao S, Hansen B, et al. Cloning of omentin, a new adipocytokine from omental fat tissue in humans. Diabetes 2003;52(Suppl 1):1-A730.

15. Shibata R, Ouchi N, Murohara T. [Omentin and cardiovascular disease]. Nihon Yakurigaku Zasshi. 2016 Mar;147(3):139-42. Japanese. doi: $10.1254 /$ fpj.147.139.

16. Du Y, Ji Q, Cai L, Huang F, Lai Y, Liu Y, et al. Association between omentin-1 expression in human epicardial adipose tissue and coronary atherosclerosis. Cardiovasc Diabetol. 2016 28;15:90 doi: 10.1186/s12933-016-0406-5.

17. Tao S, Huang $Y Q$, Cai AP, Huang C, Zhang $Y$, Tang ST, et al. Association of Serum Omentin-1 Concentrations with the Presence of Atrial Fibrillation. Med Sci Monit. 2016;22:4749-4754. doi: 10.12659/ msm.898202.

18. Mazurek T, Kiliszek M, Kobylecka M, Skubisz-Głuchowska J, Kochman J, Filipiak K, et al. Relation of proinflammatory activity of epicardial adipose tissue to the occurrence of atrial fibrillation. Am J Cardiol. 2014;113(9):1505-8. doi: 10.1016/j.amjcard.2014.02.005.

19. Guo Y, Lip GY, Apostolakis S. Inflammation in atrial fibrillation. J Am Coll Cardiol. 2012;60(22):2263-70. doi: 10.1016/j.jacc.2012.04.063.

20. Kremen J, Dolinkova M, Krajickova J, Blaha J, Anderlova K, Lacinova $Z$, et al. Increased subcutaneous and epicardial adipose tissue production of proinflammatory cytokines in cardiac surgery patients: possible role in postoperative insulin resistance. J Clin Endocrinol Metab. 2006;91(11):4620-7. doi: 10.1210/jc.2006-1044.

21. Mazurek T, Zhang L, Zalewski A, Mannion JD, Diehl JT, Arafat H,et al. Human epicardial adipose tissue is a source of inflammatory mediators. Circulation. 2003;108(20):2460-6 doi: 10.1161/01. CIR.0000099542.57313.C5

22. Fan $\mathrm{Y}, \mathrm{Zhao} \mathrm{X}, \mathrm{Li} \mathrm{X}, \mathrm{Li} \mathrm{N}, \mathrm{Hu} \mathrm{X}$. Cardiac troponin and adverse outcomes in atrial fibrillation: A meta-analysis. Clin Chim Acta. 2018 Feb;477:48-52. doi: 10.1016/j.cca.2017.11.040.

23. Roever L, Resende ES, Roerver-Borges AS. Impact of pro-atrial natriuretic peptide in atrial fibrillation and stroke. Eur J Prev Cardiol. 2017 Aug;24(12):1239-1241. doi: 10.1177/2047487317707832. 
24. Pan J, Wang W, Wu X, Kong F, Pan J, Lin J,et al. Inflammatory cytokines in cardiac pacing patients with atrial fibrillation and asymptomatic atrial fibrillation. Panminerva Med. 2018 Sep;60(3):8691. doi: 10.23736/S0031-0808.18.03452-3.

25. Fain JN, Sacks HS, Buehrer B, Bahouth SW, Garrett E, Wolf RY, et al. Identification of omentin mRNA in human epicardial adipose tissue: comparison to omentin in subcutaneous, internal mammary artery periadventitial and visceral abdominal depots. Int J Obes (Lond). 2008;32(5):810-5. doi: 10.1038/sj.ijo.0803790.
26. Bays, H.E. Adiposopathy is "sick fat" a cardiovascular disease?. J Am Coll Cardiol. 2011;57(25):2461-2473. doi: 10.1016/j.jacc.2011.02.038.

27. Yamawaki, H. Kuramoto, J. Kameshima, S. Usui, T. Okada, M. ve Hara Y. Omentin, a novel adipocytokine inhibits TNF-induced vascular inflammation in human endothelial cells. Biochem Biophys Res Commun. 2011;408(2): 339-343. doi: 10.1016/j.bbrc.2011.04.039 